\title{
The Prevalence of Human Bocavirus, Human Coronavirus-NL63, Human Metapneumovirus, Human Polyomavirus KI and WU in Respiratory Tract Infections in Kuwait
}

\author{
Sahar Essa ${ }^{\mathrm{a}}$ Abdullah Owayed $^{\mathrm{b}}$ Haya Altawalah $^{\mathrm{a}}$ Mousa Khadadah $^{\mathrm{c}}$ \\ Nasser Behbehanic ${ }^{c}$ Widad Al-Nakib $^{a}$ \\ Departments of ${ }^{\mathrm{a}}$ Microbiology, ${ }^{\mathrm{b}}$ Pediatrics and ${ }^{\mathrm{c}}$ Medicine, Faculty of Medicine, Kuwait University, Safat, Kuwait
}

\section{Key Words}

Respiratory tract infections . Newly discovered viruses.

Polymerase chain reaction

\begin{abstract}
Objective: The aim of this study was to investigate the prevalence of human coronavirus (HCoV)-NL63, human metapneumovirus (hMPV), human bocavirus (Boca), human polyomavirus KI (KIV) and human polyomavirus WU (WUV) in respiratory tract infections (RTI) in Kuwait. Materials and Methods: Respiratory samples from 735 hospitalized patients with RTI from September 2010 to April 2013 were evaluated for the presence of HCoV-NL63, hMPV, Boca, KIV and WUV using molecular assays, polymerase chain reaction (PCR) and reverse-transcription PCR. Results: Of the 735 patients, 285 (38.8\%) were diagnosed with viral RTI. The distribution of respiratory viruses was hMPV: 15 (5.3\%), Boca: 14 (4.9\%), WUV: 10 (3.5\%) and KIV: 4 (1.4\%). HCoV-NL63 was not detected in any of the samples. Conclusions: These newly discovered viruses were associated with the development of RTI in Kuwait. The rapid identification of these viral infections could aid in the control of nosocomial transmission, reduce the use of antibiotics and improve treatment and management strategies.

(c) 2015 S. Karger AG, Basel
\end{abstract}

\section{Introduction}

Viruses are the major causative agents of respiratory tract infections (RTI), but the etiology of many suspected cases of such infections remain unknown. This was the case in our previous study [1] where $72 \%$ of the cases of RTI remained uncharacterized. Since 2001, several new human viruses have been identified in respiratory samples [2-10]. These viruses were originally isolated from individuals with respiratory tract disease, most of whom were children. Accumulating data indicate that these viruses can cause both upper RTI (URTI) and lower RTI (LRTI) in children [2-10]. Newly discovered viruses, such as human metapneumovirus (hMPV) $[2,3]$, human coronavirus (HCoV)-NL63 [4, 5], human bocavirus (Boca) $[6,7]$, human polyomavirus KI (KIV) and human polyomavirus WU (WUV) [8-10] may be responsible for many respiratory tract illnesses, the cause of which has remained a mystery for decades.

The hMPV was originally isolated from the respiratory tracts of young children with respiratory disease [2]. Since then, the virus has been identified worldwide, and many studies have demonstrated that it is a significant cause of both URTI and LRTI in infants, young children, the elderly and immunocompromised populations [3].

\begin{tabular}{ll}
\hline KARGER 125:s & $\begin{array}{l}\text { () 2015 S. Karger AG, Basel } \\
1011-7571 / 15 / 0244-0382 \$ 39.50 / 0 \quad \text { Karger }\end{array}$ \\
$\begin{array}{l}\text { E-Mail karger@karger.com } \\
\text { www.karger.com/mpp }\end{array}$ & $\begin{array}{l}\text { This is an Open Access article licensed under the terms of the } \\
\text { Creative Commons Attribution-NonCommercial 3.0 Un- } \\
\text { ported license (CC BY-NC) (www.karger.com/OA-license), } \\
\text { applicable to the online version of the article only. Distribu- } \\
\text { tion permitted for non-commercial purposes only. }\end{array}$
\end{tabular}

Dr. Sahar Sultan Essa

Department of Microbiology

Faculty of Medicine, Kuwait University

PO Box 24923, Safat 13110 (Kuwait)

E-Mail sahar@hsc.edu.kw 
The hMPV can cause severe infections, such as bronchiolitis and pneumonia, and it is responsible for $2 \%$ of the hospitalizations of children suffering from acute RTI [3]. A study in Kuwait in 2011 indicated that the prevalence of hMPV was $5.4 \%$ [11].

In 2004, Fouchier et al. [4] and van der Hoek et al. [12] independently reported the recovery of a novel $\mathrm{HCoV}$ from a 7-month-old girl with coryza, conjunctivitis, fever and bronchiolitis. This virus, HCoV-NL63, is phylogenetically related to $\mathrm{HCoV}-229 \mathrm{E}$. Similar to $\mathrm{HCoV}-229 \mathrm{E}$, HCoV-NL63 has a worldwide distribution and has been associated with mild URTI and severe LRTI. HCoVNL63 may be responsible for $1-10 \%$ of RTI and is associated with croup [5].

In 2005, Allander et al. [6] reported the discovery of another novel human parvovirus, Boca, also isolated from respiratory specimens from children with respiratory tract disease. Since then, several studies $[7,13]$ have reported the prevalence of Boca infection worldwide as ranging from 2 to $21.5 \%$, mainly in children younger than 3 years of age in whom the virus has been associated with URTI and LRTI $[7,13]$. These reports suggest that Boca might be associated with upper and lower respiratory diseases throughout the world [7, 13]. In 2007, another two novel human polyomaviruses, KIV and WUV, were described by independent groups in Sweden and the USA $[8,9]$. To date, preliminary data show that both KIV and WUV can be detected in up to $4.5 \%$ of respiratory samples obtained from patients with acute RTI $[8,10]$.

In 2010 in Kuwait, Khadadah et al. [1] screened samples for a wide range of respiratory viruses such as respiratory syncytial virus (RSV), influenza A virus (Flu A), parainfluenza virus (PIV), adenovirus (AdV), HCoVOC43, HCoV-229E and human rhinovirus (HRV); however, $72 \%$ of the samples were negative, which suggested the potential presence of other viral etiological agents. The clinical impact of these newly discovered viruses remains unknown. The reported data clearly show that hMPV has been circulating for at least 50 years and has a definite clinical impact [2]. In addition, there is evidence to suggest that Boca is pathogenic [14], but data from previous studies suggest that WUV and KIV on their own are not pathogenic [15]. The newly discovered $\mathrm{HCoV}-\mathrm{OC} 43$ and $\mathrm{HCoV}-\mathrm{NL} 63$ were previously considered to be pathogenic $[4,5]$. We aimed here to study the prevalence of the newly discovered viruses hMPV, HCoV-NL63, Boca, KIV and WUV in RTI in Kuwait.

Newly Discovered Viruses in Respiratory Infections

\section{Materials and Methods}

\section{Study Population}

The study included 735 patients, between 3 days and 76 years of age, with URTI or LRTI, who were admitted to Mubarak Al-Kabeer Hospital, Kuwait. These patients were screened during the period from September 2010 to April 2013. The URTI included those of the throat (pharyngitis), nasopharynx (nasopharyngitis), sinuses (sinusitis), larynx (laryngitis) and trachea (tracheitis). World Health Organization criteria for LRTI in children were used [16], and American Thoracic Society criteria were used for LRTI in adults [17, 18]. The specimens were collected in the hospital and processed in the Virology Unit, Faculty of Medicine, Kuwait University. The majority of samples were collected during autumn and winter. Autumn in Kuwait occurs between September and November and winter between December and March. To detect the presence of viral nucleic acids, polymerase chain reaction (PCR) techniques were used.

Clinical Samples

The samples included bronchoalveolar lavage (BAL), tracheal aspirate (TA), nasopharyngeal aspirate (NPA) and nasopharyngeal swab (NPS). All of the specimens were collected after obtaining written informed consent from the adult patients and the parents of the underage patients (441 were $<2$ years of age and 294 were $\geq 2$ years of age). Ethical permission to perform this research study was granted by the Health Science Center and Kuwait Institute for Medical Specialization (KIMS) Joint Committee for the Protection of Human Subjects in Research.

\section{Multiplex PCR for the Detection of Respiratory Viruses}

Nucleic acid isolation and multiplex PCR were performed for respiratory viruses such as Flu A, PIV-1, PIV-2, PIV-3, RSV, $\mathrm{HCoV}-\mathrm{OC} 43, \mathrm{HCoV}-229 \mathrm{E}$ and $\mathrm{HRV}$ as previously described [1].

\section{PCR and Reverse-Transcription PCR for the Detection of}

Newly Discovered Respiratory Viruses

Viral nucleic acid was extracted from $140-\mu$ l respiratory specimens using the QIAamp Viral RNA mini kit (Qiagen, Hilden, Germany) according to the manufacturer's instructions. RNA was then eluted in $60 \mu \mathrm{l}$ of the buffer provided in the kit and stored at $-70^{\circ} \mathrm{C}$ until processing. Upon the extraction of nucleic acids from the clinical specimens, the presence of viral nucleic acids from hMPV, HCoV-NL63, Boca, KIV and WUV was determined with the primers described before $[3,5,7,10]$.

\section{PCR and Reverse-Transcription PCR Conditions}

The reverse-transcription step was performed as described by Khadadah et al. [1]. The PCR results were confirmed as previously described using a hybridization technique. The specificity of the PCR was established for each PCR format using a panel of ATCC reference viruses to check for cross-reactivity to old respiratory viruses. DNA templates (110-140 bp, Thermo Scientific) encompassing the annealing sites of the primers and probes were used as positive controls for the detection of $\mathrm{HCoV}-\mathrm{NL} 63, \mathrm{hMPV}$, Boca, WUV and KIV.

\section{Statistical Analysis}

Data analysis was performed using the Statistical Package for the Social Sciences software (SPSS v20.0; IBM Corp, Armonk, N.Y., USA). Descriptive statistics for continuous variables were 
compared using the nonparametric Mann-Whitney $U$ test or the Kruskal-Wallis test. For categorical variables, the $\chi^{2}$ test, the Fisher exact test or the $\mathrm{Z}$ test was applied to evaluate the difference between proportions or to assess whether there were any associations between the proportions. A two-tailed probability value $\mathrm{p}<$ 0.05 was considered statistically significant.

\section{Results}

Of the 735 patients, 285 (38.8\%) displayed evidence of viral infection, 163 (57.2\%) of whom were males and 122 (42.8\%) were females. Among the 285 patients, 331 viruses were detected because $44(15.4 \%)$ patients were coinfected with two respiratory viruses and $2(0.7 \%)$ were coinfected with three respiratory viruses (table 1 ). The most common viral infections were HRV 122 (42.8\%), followed by Flu A 47 (16.5\%), RSV 42 (14.7\%), HCoVOC43 25 (8.8\%), AdV 22 (7.7\%) and hMPV 15 (5.3\%). The newly discovered viruses WUV, KIV, Boca and hMPV were detected in $43(15.1 \%)$ of the patients with RTI (table 1).

Of the 285 patients, 167 (58.6\%) respiratory viral infections were detected in infants aged $<2$ years (table 2). Among these infections, Boca was responsible for 11 (3.9\%), hMPV for 7 (2.5\%) and KIV for 3 (1.1\%). Regarding the other viruses, HRV was responsible for 64 (22.5\%), RSV for $36(12.6 \%)$ and $\mathrm{HCoV}$-OC43 for 11 (3.9\%) infections. It was interesting to note that $20(7 \%)$ of the Flu A infections were in patients aged $>30$ years. Overall, the majority of RTI, reaching 202 (70.9\%), were among children $\leq 5$ years of age (table 2 ).

The distribution of viruses detected in relation to hospital admission is shown in table 2 . The majority of the patients admitted to wards had HRV infections $(\mathrm{n}=68$, 23.9\%), followed by patients with Flu A infections ( $\mathrm{n}=35$, $12.3 \%)$. Most of the children were admitted to the pediatric intensive care unit for HRV ( $\mathrm{n}=43,15.1 \%)$, followed by RSV ( $n=23,8.1 \%)$. Furthermore, $11(3.9 \%)$ of the patients with viral respiratory infection were admitted to the intensive care unit due to HRV, followed by Flu A ( $n=6,2.1 \%$ ).

These viruses were most commonly detected in NPS (104 samples, 30.1\%), followed by NPA (99 samples, 28.7\%), BAL (87 samples, 25.5\%) and TA (55 samples, $15.9 \%$; table 2). Table 3 shows that the majority of the infections caused by the investigated respiratory viruses affected the LRT (251 infections, 88.1\%) rather than the URT (80 infections, 28.1\%). Pneumonia and bronchiolitis were the most frequent reasons for hospitalization (170 infections, 59.6\%).
Table 1. Frequency of the detection of 331 viruses in 285 patients with viral RTI in Kuwait

\begin{tabular}{lc}
\hline Respiratory virus & Positive, $\mathrm{n}(\%)$ \\
\hline HRV & $122(42.8)$ \\
Flu A & $47(16.5)$ \\
RSV & $42(14.7)$ \\
HCoV-OC43 & $25(8.8)$ \\
AdV & $22(7.7)$ \\
hMPV & $15(5.3)$ \\
HCoV-229E & $14(4.9)$ \\
Boca & $14(4.9)$ \\
PIV-3 & $10(3.5)$ \\
WUV & $10(3.5)$ \\
PIV-1 & $6(2.1)$ \\
KIV & $4(1.4)$ \\
HCoV-NL63 & 0 \\
PIV-2 & 0 \\
\hline Total & 331 \\
\hline
\end{tabular}

The prevalence of respiratory viral infections was highest during the period from October to February and then declined to the lowest level from July to September (fig. 1). The incidence of newly discovered viral infections was highest during the months of January, February and October. Positivity for WUV and hMPV peaked in February [3 (1.1\%) and 7 infections (2.5\%), respectively] and Boca peaked in March (5 infections, $1.8 \%$ ). KIV presented consistent numbers from October to January (1 infection, $0.4 \%)$. The other respiratory viral infections were generally present throughout the year, reaching a peak during the winter months from October to February.

\section{Discussion}

The age distribution in this study was comparable to that reported in a previous study [19] and the detection rate and sex distribution were similar to that reported in a study from Turkey [20].

LRTI (pneumonia, bronchiolitis, bronchopneumonia and respiratory distress) was diagnosed in $88.1 \%$ and URTI (pharyngitis and croup) in $28.1 \%$ of the hospitalized patients (table 3). Moreover, 30 (10.5\%) of the hospitalized patients infected with the newly discovered viruses suffered from LRTI, and 13 (4.6\%) suffered from URTI. Bronchiolitis was the most common clinical symptom (106 patients or $37.2 \%$ ), followed by pneumonia (64 patients or 22.5\%) (table 3). This result is similar to that described in a study in Saudi Arabia [19]. Most of the patients 
Table 2. Frequency of respiratory viruses in relation to sex, age, hospital admission and the type of specimen collected

\begin{tabular}{|c|c|c|c|c|c|c|c|c|c|c|c|c|c|}
\hline & WUV & KIV & Boca & hMPV & AdV & Flu A & RSV & PIV-1 & PIV-3 & HRV & HCoV-OC43 & HCoV-229E & Total \\
\hline Female & $4(1.4)$ & $1(0.4)$ & $6(2.1)$ & $7(2.5)$ & $9(3.2)$ & $19(6.6)$ & $17(6)$ & $3(1.1)$ & $4(1.4)$ & $53(18.6)$ & $12(4.2)$ & $6(2.1)$ & $141(49.5)^{1}$ \\
\hline$<2$ years & $2(0.7)$ & $3(1.1)$ & $11(3.9)$ & $7(2.5)$ & $10(3.5)$ & $13(4.6)$ & $36(12.6)$ & $2(0.7)$ & $5(1.8)$ & $64(22.5)$ & $11(3.9)$ & $3(1.5)$ & $167(58.6)^{1}$ \\
\hline $2-5$ years & $2(0.7)$ & - & $2(0.7)$ & $1(0.4)$ & $3(1.1)$ & $10(3.5)$ & - & $1(0.4)$ & - & $12(4.2)$ & $2(0.7)$ & $2(0.7)$ & $35(12.3)^{1}$ \\
\hline $6-10$ years & $2(0.7)$ & - & - & $1(0.4)$ & $4(1.4)$ & $1(0.4)$ & $2(0.7)$ & - & - & $13(4.6)$ & $5(1.8)$ & - & $28(9.8)^{1}$ \\
\hline $11-30$ years & $2(0.7)$ & - & - & $4(1.4)$ & $2(0.7)$ & $3(1.1)$ & $1(0.4)$ & - & - & $6(2.1)$ & - & $3(1.1)$ & $21(7.4)^{1}$ \\
\hline ICU & $1(0.4)$ & - & $1(0.4)$ & $1(0.3)$ & $2(0.7)$ & $6(2.1)$ & $2(0.7)$ & $2(0.7)$ & $2(0.7)$ & $11(3.9)$ & $4(1.4)$ & $3(1.1)$ & $35(12.3)^{1}$ \\
\hline PICU & $3(1.1)$ & - & $6(2.1)$ & $4(1.4)$ & $8(2.8)$ & $6(2.1)$ & $23(8.1)$ & $1(0.4)$ & $3(1.1)$ & $43(15.1)$ & $4(1.4)$ & - & $101(35.4)^{1}$ \\
\hline NPS & $3(30)$ & $2(50)$ & $4(28.6)$ & $4(26.7)$ & $7(2.5)$ & $10(3.5)$ & $11(3.9)$ & $3(1.1)$ & $3(1.1)$ & $43(15.1)$ & $5(1.8)$ & $4(1.4)$ & $104(30.1)^{2}$ \\
\hline NPA & $4(40)$ & $1(25)$ & $6(42.8)$ & $5(33.3)$ & $8(2.8)$ & $15(5.3)$ & $9(3.2)$ & $1(0.4)$ & $4(1.4)$ & 33 (11.6) & $5(1.8)$ & $3(1.1)$ & $99(28.7)^{2}$ \\
\hline BAL & $2(20)$ & - & $2(14.3)$ & $4(26.7)$ & $5(1.8)$ & $15(5.3)$ & $14(4.9)$ & $1(0.4)$ & $2(0.7)$ & $27(9.5)$ & $10(3.5)$ & $4(1.4)$ & $87(25.2)^{2}$ \\
\hline $\mathrm{TA}$ & $1(10)$ & $1(25)$ & $2(14.3)$ & $2(13.3)$ & $2(0.7)$ & $7(2.5)$ & $8(2.8)$ & $1(0.4)$ & $1(0.4)$ & $19(6.7)$ & $5(1.8)$ & $3(1.1)$ & $55(15.9)^{2}$ \\
\hline
\end{tabular}

Figures denote $\mathrm{n}(\%)$. ICU $=$ Intensive care unit; PICU $=$ pediatric intensive care unit.

${ }^{1}$ The number in parentheses represents the number of infections in relation to the total number of patients $(\mathrm{n}=285)$ in $\%$.

${ }^{2}$ The number in parentheses represents the number of infections in relation to the total number of samples $(n=345)$ in $\%$.

Table 3. Frequency of respiratory viruses in 285 patients in relation to symptom presentation

\begin{tabular}{|c|c|c|c|c|c|c|c|c|c|c|c|c|c|}
\hline & WUV & KIV & Boca & hMPV & $\mathrm{HCoV}-\mathrm{OC} 43$ & $\mathrm{HCoV}-229 \mathrm{E}$ & AdV & Flu A & RSV & PIV-1 & PIV-3 & HRV & Total \\
\hline Bronchiolitis & $5(1.8)$ & $1(0.4)$ & $8(2.8)$ & $3(1.1)$ & $6(2.1)$ & $3(1.1)$ & $3(1.1)$ & $10(3.5)$ & $20(7)$ & $1(0.4)$ & $2(0.7)$ & $44(15.4)$ & $106(37.2)$ \\
\hline Respiratory distress & $1(0.4)$ & $1(0.4)$ & $3(1.1)$ & $3(1.1)$ & $2(0.7)$ & $5(1.5)$ & $4(1.4)$ & $5(1.8)$ & $3(1.1)$ & - & $2(0.7)$ & $22(7.7)$ & $51(17.9)$ \\
\hline Total LRTI & $7(2.5)$ & $3(1.1)$ & $12(4.2)$ & $8(2.8)$ & $20(7.0)$ & $12(4.2)$ & $14(4.9)$ & $35(12.2)$ & $32(11.2)$ & $3(1.1)$ & $6(2.1)$ & $99(34.7)$ & $251(88.1)$ \\
\hline Unspecified URTI & $3(1.1)$ & - & $1(0.4)$ & $5(1.8)$ & $3(1.1)$ & $1(0.4)$ & $7(2.5)$ & 8( & - & - & $2(0.7)$ & $17(6)$ & $47(16.5)$ \\
\hline Total URTI & $3(1.1)$ & $1(0.4)$ & $2(0.7)$ & $7(2.5)$ & $5(1.8)$ & $2(0.7)$ & $8(2.8)$ & $12(4.2)$ & $10(3.5)$ & $3(1.1)$ & $4(1.4)$ & $23(8)$ & $80(28.1)$ \\
\hline
\end{tabular}

The number in parentheses represents the number of infections in relation to the total number of patients $(\mathrm{n}=285)$ in \%.

with respiratory virus infections in this study were infants $<2$ years of age, pointing to this being the age group that is the most affected, similar to previous studies $[1,19,20]$.

HRV was found to be the major cause of viral respiratory infections in this study $(42.8 \%)$, confirming that HRV is the major cause of RTI as in other countries in the Gulf region [21] and other parts of the world [22]. HCoVOC43 was detected in 25 patients (8.8\%); this is within the range of 3.3-11.1\% reported in another hospitalized cohort [23]. Among the newly discovered viruses, the 5.3\% of hMPV meant it was the most commonly detected virus, followed by $4.9 \%$ of Boca, also similar to the results of a previous study [11].

Respiratory viruses were detected mostly in NPS samples (30.1\% or 104 samples) from a total of 345 samples, followed by samples of NPA ( $28.7 \%$ or 99 samples), BAL (25.2\% or 87 samples) and TA ( $15.9 \%$ or 55 samples; table 2).

The prevalence of respiratory viral infections was highest during the period from October to February and then declined to the lowest level from July to September (fig. 1). The incidence of newly discovered viral infections was highest during the months of January, February and October. The peak season for WUV $(1.1 \%, \mathrm{n}=3)$ and hMPV $(2.5 \%, \mathrm{n}=7)$ was in February, and the peak season for Boca occurred in March $(1.8 \%, \mathrm{n}=5)$. The positivity for KIV was consistent from October to January $(0.4 \%$, $\mathrm{n}=1$ ). The other respiratory viral infections were generally present throughout the year, reaching a peak during the winter months from October to February. 


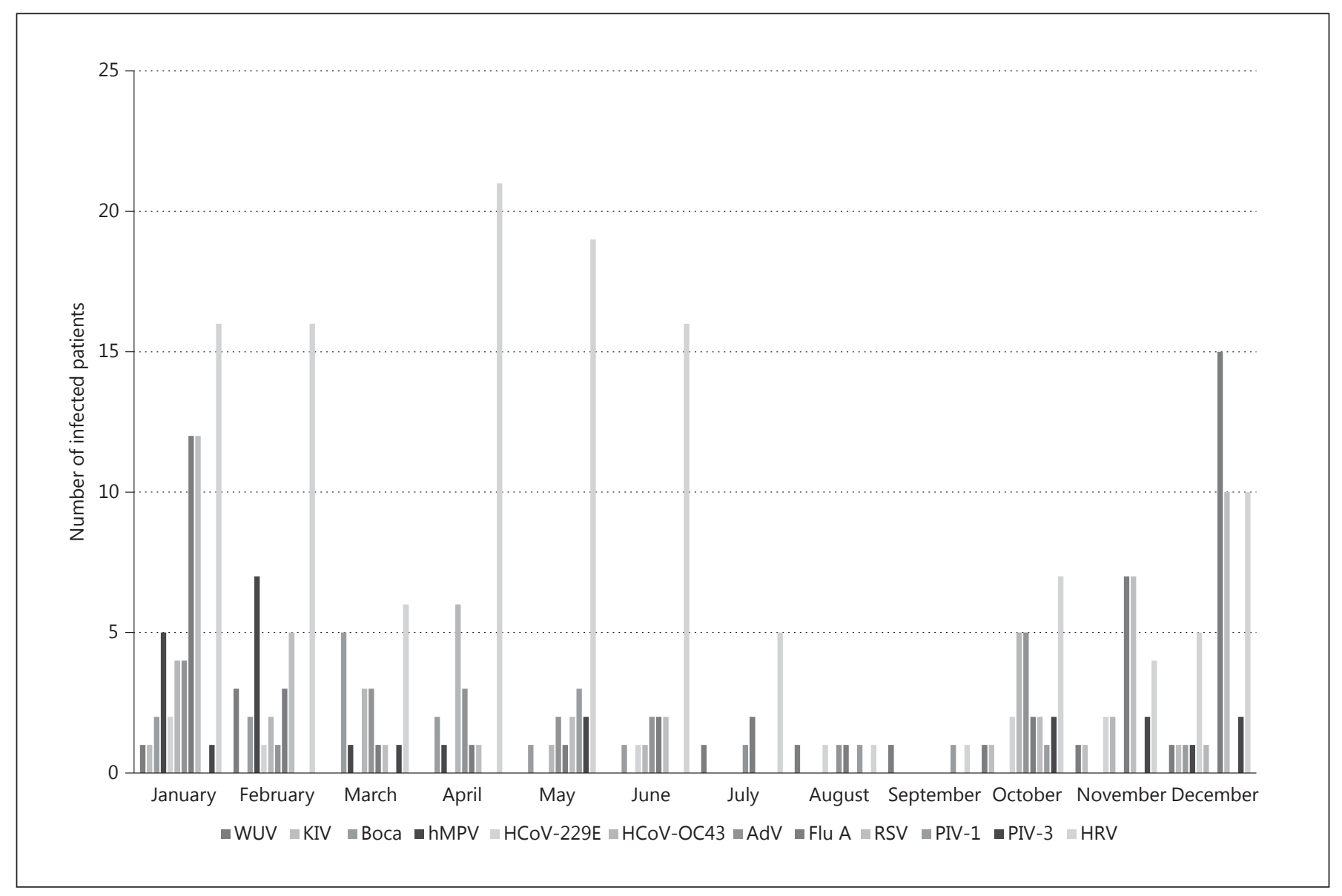

Fig. 1. Monthly distribution of respiratory viruses in Kuwait.

This seasonal distribution of respiratory viral infections during the period from October 2010 to February 2013 indicates a high incidence in the winter months and a low incidence in spring and summer. Previous studies showed that hMPV infections appear to have a seasonal distribution. In the Philippines, peak positivity for hMPV infections occurred in March [22]. In Hong Kong, hMPV outbreaks were apparent during the spring/summer season [24]. In contrast, in Norway, hMPV outbreaks occurred during the winter season [25]. Other reports have suggested that hMPV may be circulating throughout the year [26]. Data from our study showed that hMPV infections appeared to have a seasonal distribution in Kuwait. The peak months were January and February, which indicated that hMPV was prevalent in the winter. A seasonal distribution of Boca was noted from December to June, especially during the winter/spring season, which is consistent with other studies [6,27]. However, in China, the peak seasons were noted as being from June to No- vember, especially during the summer season [28]. In Canada, Boca activity did not seem to be associated with a specific time of the year [29]. A seasonal distribution of WUV was noted in summer, autumn and winter. KIV activity was detected only during the autumn/winter months from October to January. In contrast, in Japan, the peak seasonal activity of WUV and KIV was detected during the spring/early summer months [30]. This variety of reported seasonal distributions of WUV, KIV, hMPV and Boca infections suggests the presence of other factors that may affect the activity of these viruses throughout the year in different countries, such as temperature, humidity and precipitation.

A general assessment of seasonality and age distribution, especially conclusions based on epidemiological data, will be useful for medical decision-making, particularly decisions related to the prescription of antiviral treatments, the limitation of antibiotic therapy and the application of control policies for infections. 


\section{Conclusion}

These newly discovered viruses were associated with the development of RTI in Kuwait. Hence, the rapid identification of these viral infections could aid in the control of nosocomial transmission, reduce the use of antibiotics and improve treatment and management strategies.

\section{Acknowledgments}

This research was funded by the Research Sector, Kuwait University, grant No. MI 03/08. We are especially thankful to Dr. Wassim Chehadeh, Associate Professor, Microbiology Department, Faculty of Medicine, for his excellent technical assistance in the viral analysis by PCR.

\section{References}

1 Khadadah, M, Essa S, Higazi Z, et al: Respiratory syncytial virus and human rhinoviruses are the major causes of severe lower respiratory tract infections in Kuwait. J Med Virol 2010;82:1462-1467.

2 van den Hoogen BG, de Jong JC, Groen J, et al: A newly discovered human pneumovirus isolated from young children with respiratory tract disease. Nat Med 2001;7:719-724.

-3 Williams JV, Harris PA, Tollefson SJ, et al: Human metapneumovirus and lower respiratory tract disease. N Engl J Med 2004;350: 443-450.

4 Fouchier RA, Hartwig NG, Bestebroer TM, et al: A previously undescribed coronavirus associated with respiratory disease in humans. Proc Natl Acad Sci USA 2004;101:6212-6216.

5 Pyrc K, Berkhout B, van der Hoek L: The novel human coronaviruses NL63 and HKU1. J Virol 2007;81:3051-3057.

-6 Allander T, Tammi MT, Eriksson M, et al: Cloning of a human parvovirus by molecular screening of respiratory tract samples. Proc Natl Acad Sci USA 2005;102:12891-12896.

-7 Allander T, Jartti T, Gupta S, et al: Human bocavirus and acute wheezing in children. Clin Infect Dis 2007;44:904-910.

-8 Allander T, Anderson K, Gupta S, et al: Identification of a third human polyomavirus. J Virol 2007;81:4130-4136.

-9 Gaynor AM, Nissen MD, Whiley DM, et al: Identification of a novel polyomavirus from patients with acute respiratory tract infections. PLoS Pathog 2007;4:e64.

10 Bialasiewicz S, Whiley DM, Lambert SB, et al: Development and evaluation of real-time PCR assays for the detection of the newly identified $\mathrm{KI}$ and $\mathrm{WU}$ polyomaviruses. J Clin Virol 2007;40:9-14.
11 Al-Turab M, Chehadeh W, Al-Mulla F, et al: Human metapneumovirus in patients with respiratory tract infection in Kuwait. J Med Virol 2011;83:1811-1817.

12 van der Hoek L, Pyrc K, Jebbink MF, et al: Identification of a new human coronavirus. Nat Med 2004;10:368-373.

13 Qu XW, Duan ZJ, Qi ZY, et al: Human bocavirus infection, People's Republic of China. Emerg Infect Dis 2007;13:165-168.

14 Calvo C, Garcia-Garcia ML, Pozo F, et al: Clinical characteristics of human bocavirus infections compared with other respiratory viruses in Spanish children. Pediatr Infect Dis J 2008;27:677-680.

15 Norja P, Ubillos I, Templeton K, et al: No evidence for an association between infections with WU and KI polyomaviruses and respiratory disease. J Clin Virol 2007;40:307-311.

16 World Health Organization: Consultative meeting to review evidence and research priorities in the management of acute respiratory infections. Geneva, WHO, 2003.

17 American Thoracic Society: Guidelines for the management of adults with communityacquired pneumonia. Am J Respir Crit Care Med 2001;163:1730-1754.

18 American Thoracic Society: Guidelines for the management of adults with hospital-acquired, ventilator-associated, and healthcareassociated pneumonia. Am J Respir Crit Care Med 2005;171:388-416.

19 Al-Hajjar S, Akhter J, al Jumaah S, et al: Respiratory viruses in children attending a major referral Centre in Saudi Arabia. Ann Trop Paediatr 1998;18:87-92.

20 Hatipoglu N, Somer A, Badur S, et al: Viral etiology in hospitalized children with acute lower respiratory tract infection. Turk J Pediatr 2011;53:508-516.
21 Althani A, Bushra S, Shaath N, et al: Characterisation of winter respiratory viral infections in patients with asthma and COPD in Qatar. Arch Virol 2013;158:1079-1083.

22 Suzuki A, Lupisan S, Yuki Furuse Y, et al: Respiratory viruses from hospitalized children with severe pneumonia in the Philippines. BMC Infect Dis 2012;12:267-278.

-23 Walsh EE, Shin JH, Falsey AR: Clinical impact of human coronaviruses 229E and OC43 infection in diverse adult populations. J Infect Dis 2013;208:1634-1642.

24 Peiris JS, Tang WH, Chan KH, et al: Children with respiratory disease associated with metapneumovirus in Hong Kong. Emerg Infect Dis 2003;9:628-633.

25 Dollner H, Risnes K, Radtke A, et al: Outbreak of human metapneumovirus infection in Norwegian children. Pediatr Infect Dis J 2004; 23:436-440.

26 Li XY, Chen JY, Kong M, et al: Prevalence of human metapneumovirus in hospitalized children with respiratory tract infections in Tianjin. Arch Virol 2009;154:1831-1836.

27 Manning A, Russell V, Eastick K, et al: Epidemiological profile and clinical associations of human bocavirus and other human parvoviruses. J Infect Dis 2006;194:1283-1290.

28 Chen ZR, Mize M, Wang YQ, et al: Clinical and epidemiological profiles of lower respiratory tract infection in hospitalized children due to human bocavirus in a subtropical area of China. J Med Virol 2014;86:2154-2162.

29 Bastien N, Brandt K, Dust K, et al: Human bocavirus infection, Canada. Emerg Infect Dis 2006;12:848-850.

30 Okada M, Hamada H, Sato-Maru H, et al: WU polyomavirus detected in respiratory tract specimens from young children in Japan. Pediatr Int 2013;55:536-537. 